\title{
Critical Care Ultrasonography and Its Application for COVID-19
}

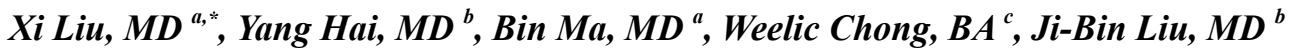 \\ ${ }^{a}$ Department of Ultrasound, Air Force Medical Center, Air Force Medical University, Beijing, China; ${ }^{b}$ Department of Radiology, Thomas \\ Jefferson University, Philadelphia, PA, USA; ${ }^{c}$ Sidney Kimmel Medical College, Thomas Jefferson University, Philadelphia, PA, USA \\ Received April 11, 2020; revision received April 13, 2020; accepted April 14, 2020.
}

\begin{abstract}
Ultrasound has developed as an invaluable tool in diagnosis and proper management in the intensive care unit (ICU). Application of critical care ultrasonography is quite distinct from the routine comprehensive diagnostic ultrasound exam, because the urgent setting mandates a goal-directed approach. Performing accurate and efficient critical care ultrasound requires ultrasound providers to first understand the pathophysiology of the disease and related imaging findings, and then follow the protocols to perform a focused ultrasound exam. In the ongoing coronavirus disease 2019 (COVID-19) pandemic, ultrasound plays an essential role in diagnosing and monitoring critically ill COVID-19 patients in the ICU. Our review focuses on the basics and clinical application of critical care ultrasound in diagnosing common lung disease, COVID-19 pulmonary lesions, pediatric COVID-19, and cardiovascular dysfunction as well as its role in ECMO and interventional ultrasonography.
\end{abstract}

Key words: Critical care; Ultrasonography; COVID-19; Lung; Heart; Kidney; Vascular

Advanced Ultrasound in Diagnosis and Therapy 2020;02:043-049

DOI: $10.37015 / A U D T .2020 .200035$

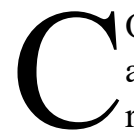
OVID-19 is an infectious disease caused by a novel coronavirus known as severe acute respiratory syndrome coronavirus 2 (SARSCoV-2). The disease has been declared a pandemic by World Health Organization (WHO) due to its highly contagious nature, rapid transmission, swift clinical course, profound worldwide impact, and high mortality among critically ill patients. Like previous coronavirus epidemics (SARS and MERS) infection from coronaviruses family, SARS-CoV-2 mainly cause pulmonary damage like pneumonia and acute respiratory distress syndrome (ARDS), which may further lead to multi-organ failure and disseminated intravascular coagulation (DIC) in certain groups of patients [1-4]. Some patients with mild symptoms can rapidly and unexpectedly become critically ill.

Radiology departments play a crucial role in early diagnosis, appropriate management, and close followup of COVID-19 patients. Although chest computerized tomography $(\mathrm{CT})$ and chest x-ray are currently the main imaging modality of evaluating COVID-19, ultrasound has a role in the course of COVID-19 prompt diagnosis and treatment due to its portability and non-invasiveness. In the intensive care unit (ICU), ultrasound could be effectively performed at bedside to avoid transferring a sick patient out of the ICU which would delay proper treatment [5]. In addition, the bedside real-time ultrasound evaluation of multi organ systems can be repeated as many times as necessary, which is helpful to timely monitor the dynamic disease course. The clinical value of using ultrasound in ICU for COVID-19 patients has been proven [6].

In China, ultrasound has shown promise for helping to monitor patients with COVID-19, especially in ICU settings. Beyond pulmonary evaluation, ultrasound can also evaluate multiple organs and systems that may be affected in patients with COVID-19, including heart, blood vessels, and kidneys, etc. [3,6-9]. Further, ultrasound examination in the ICU is goal-directed, rapid and focused. Our review focuses on the basics and clinical application of critical care ultrasound in diagnosing common lung disease, COVID-19 pulmonary

\footnotetext{
* Corresponding author: Department of Ultrasound, Air Force Medical Center, Air Force Medical University, Beijing, China e-mail: liuxi2007@fmmu.edu.cn unrestricted use, distribution and reproduction in any medium provided that the original work is properly attributed.
} 
lesions, pediatric COVID-19, and cardiovascular dysfunction as well as its role in ECMO and interventional ultrasonography.

\section{Physiology Basics of Pulmonary Ultrasonography}

Lung ultrasonography is based on the analysis of echogenic sonography and the differentiation of artifacts. The ultrasound imaging of different pulmonary pathologies is mainly determined by the "gas-liquid ratio" of lung parenchyma and interstitial tissue. Different pathological tissues have different gas-liquid ratios, resulting in different images, which could be further categorized into:

(1) normal A-line: the lung tissue with normal ventilation will show transverse artifacts (A-line) parallel to the pleural line. The pleural line is the echo reflection of the pleura formed by the interface of the pleural lung surface. It is smooth and uniform in normal condition, with the thickness of less than $0.5 \mathrm{~mm}$. The A-line is parallel to and equidistant from the pleura, and the distance between the pleural line and the A-line is equal to the distance between the skin and the pleural line (Fig. 1). The appearance of A-line in a lung region means that the area under inspection is inflated normally. In this normal situation, the gas-liquid ratio is about 0.98 [2].
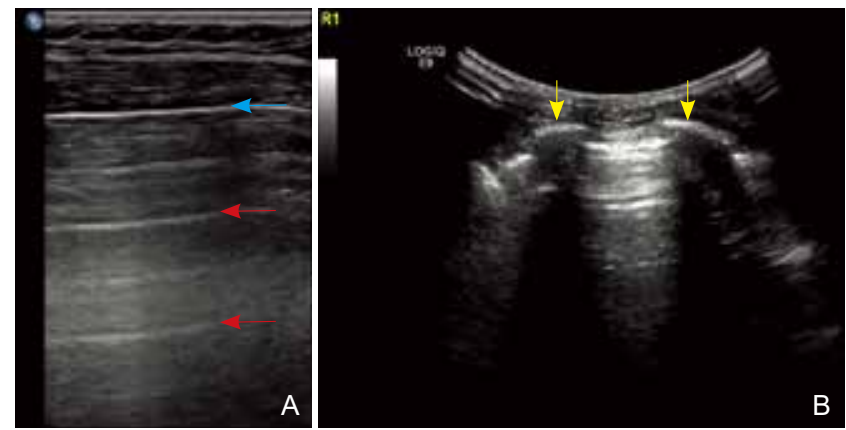

Figure 1 (A) Normal lung sonography by linear probe. The pleura line is a linear reflection formed by the interface echo between pleura and lung surface (blue arrow). A-line (red arrow) is parallel to and equidistant from the pleura line, and the distance between the pleural line and the A-line is equal to the distance between the skin and the pleural line. (B) Intercostal longitudinal section by convex array probe shows hyperechoic upper and lower ribs and the shadow behind (yellow arrow), the linear hyperechoic pleural line in between. Together with the parallel A-line artifacts beneath pleural line, a "bat sign" is displayed.

(2) The lung tissue with increased liquid content and partial ventilation will form a longitudinal artifact (B-line), which radiates perpendicularly from the pleural line (Fig. 2). The B-line has long been recognized as the sign of pulmonary interstitial syndrome [10]. In this situation, the gas-liquid ratio is about 0.95 .

(3) A further increase in liquid content will lead to complete disappearance of gas, making the sonogram of lung tissue look similar to solid organs like liver and spleen (hepatization) (Fig. 3). In this situation, the gas- liquid ratio is about 0.1 . As more fluid leaks out, severe pleural effusion is seen (Fig. 4), and the gas-liquid ratio could be 0 .
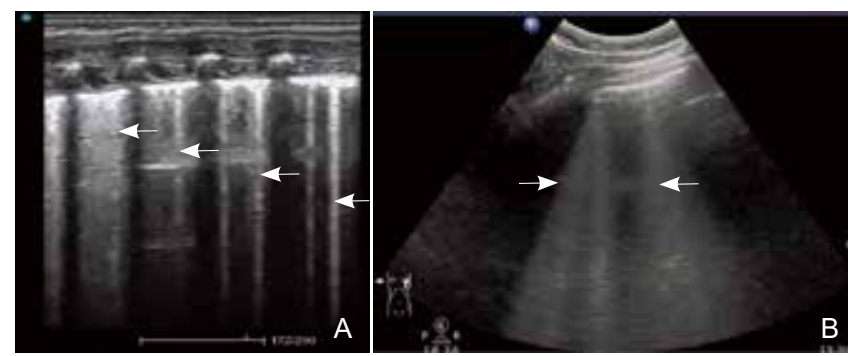

Figure 2 B-line radiates perpendicularly from the pleural line, reaching the deep echo of the lung field. (A) linear array and (B) convex array probe images show B line (arrows).
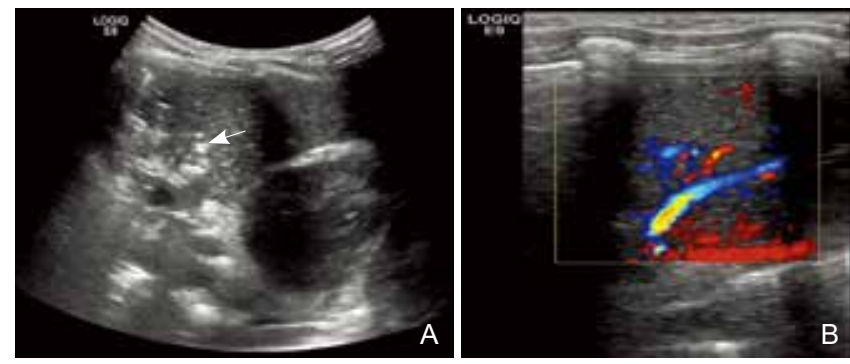

Figure 3 (A) Further increased liquid content will lead to complete disappearance of gas, making the sonography of lung tissue look similar to solid organs like liver and spleen (hepatization). The hyperechoic in the lung parenchymal is unabsorbed air in bronchi (arrows). (B) Linear high frequency probe is recommended to pediatric examination. It is helpful to show more details of solid lung (L) with blood flow distribution.

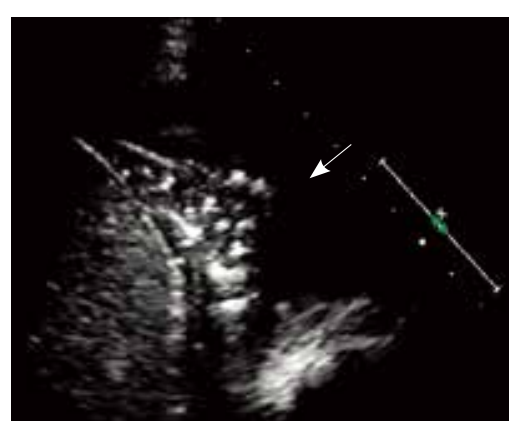

Figure 4 More fluid exudation or leaking out cause pleural effusion (arrow). Pulmonary effusion often appears in the gravity dependent area where consolidation of lung tissue can be seen in the liquid dark area with hyper echo of unabsorbed gas.

\section{Common Ultrasound Findings in Lung Disease}

For peripheral lung diseases, ultrasound can help diagnose diseases caused by altered gas-liquid ratio, including interstitial inflammation, alveolar edema, subpleural consolidation, and tumor. The degree of gasliquid ratio alteration in the lung can be categorized into normal, moderate, severe, and consolidation using semiquantitative analysis of the density and distribution of $\mathrm{B}$-lines. It is generally believed that the distance between two B-lines greater than $7 \mathrm{~mm}$ (B7 line) indicates 
interstitial edema, and the distance between B-lines less than $3 \mathrm{~mm}$ (B3 line) indicates alveolar edema [11]. In fact, it can be generally understood that the higher the density of B line, the more advanced the degree of pulmonary edema. As the disease progresses, the $\mathrm{B}$ line fusion occurs and the distance between B-lines becomes indistinguishable. With further aggravation, all the B-lines within the intercostal lung field appear hyperechoic, white and fused, and some even covering the costal acoustic shadow, which is called "white lung" (Fig. 5).
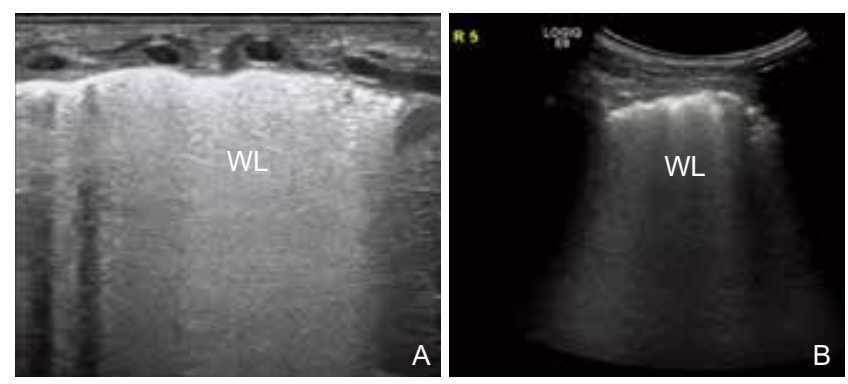

Figure 5 With further aggravation, all the B-lines within the intercostal lung field appear hyperechoic, white and fused, and some even covering the costal acoustic shadow, which is called "white lung" (WL). (A) linear array image; (B) convex array image.

\section{Ultrasound Findings of COVID-19 Pulmonary Lesions}

The SARS-CoV-2 particles enter the airway and lung by respiration, and it could reach the bronchioles and alveoli. Therefore, the COVID-19 lesions mostly distributed in the terminal pleura. The autopsy of COVID-19 lung tissue showed diffuse damage of the alveoli with mucus exudation, which further contribute to lung consolidation [12]. On CT, COVID-19 lungs usually display as ground-glass opacities on the periphery of the diseased lungs or near the pleura. Ultrasound can show the distribution of COVID-19 lesions, and also monitor the B-line density and areas of consolidation at different stages of the disease. Recently, several Chinese webinar presentations and publications have shown a high degree of consistency between ultrasound and CT findings in COVID-19 patients [13-16]. When medical resources are in shortage and CT scan cannot be implemented on a large scale during the outbreak, ultrasound can be applied for early and rapid diagnosis and triage.

\section{Common Pulmonary Ultrasound Findings In ICU}

One particular use of pulmonary ultrasound in the ICU is to diagnose acute respiratory distress syndrome (ARDS). According to the international lung ultrasound consensus [17], the following signs suggest the existence of ARDS: (1) Uneven B-line distribution; (2) Abnormal pleura line (thickening or interruption); (3) Subpleural consolidation near anterior chest wall; (4) Normal and diseased lung parenchyma co-exist; (5) Weakened or absent pulmonary gliding sign. In clinical practice, we found that the distribution of diseased area of lungs from ARDS are often gravity-dependent, that is, consolidation areas are more likely to appear in the dorsal segment of the lower lobe of both lungs.

Another use of pulmonary ultrasound in the ICU is to evaluate pulmonary edema. The differential diagnosis of cardiogenic pulmonary edema should be considered in combination with cardiac function index. If wide-spaced B-lines (B7 line) are found in all areas, echocardiography should be considered to check whether for signs of heart failure. The evaluation of heart function includes systolic and diastolic function, and the filling pressure index should also be used. One clinical shortcut is to consider echocardiography when B7 lines are found in the bilateral anterior chest wall.

In addition, cardiopulmonary combined ultrasonography and diaphragmatic ultrasound can help with recruitment maneuver, prone position ventilation, selection of autonomic respiratory intensity, and the whole process of mechanical ventilation including determining the causes of ventilation failures and the timing of ventilator wean-off. Previous studies have also discussed the application of ultrasound in these aspects [18-21].

\section{Limitations of Pulmonary Ultrasound}

There are several limitations of ultrasound: (1) Lesions can be displayed by ultrasound when the edema and consolidation reach the pleura. On the contrary, if the lesions do not reach the pleura, the center of the lesion cannot be identified and evaluated by ultrasound, making ultrasonography inaccurate for quantifying the extent and location of some pulmonary lesions compared to CT. (2) Because B-line and consolidation are frequentlyappearing signs in various lung diseases, the specificity of using ultrasound alone for diagnosis is not high. Thus, an accurate diagnosis often needs information from other imaging modality, clinical manifestations, and laboratory tests. (3) Conditions like obesity and subcutaneous emphysema will cause significant sound attenuation, which affects the image quality, especially for deep lesions. (4) Any overlap with scapula, rib, or dressings can cause blind area of sonography. (5) Ultrasound is highly operator-dependent. The experience of the operator and the cooperation with clinicians will affect the interpretation of ultrasound images. 


\section{Role of Ultrasound in Pediatric COVID-19 Patients}

Ultrasound is a very important imaging modality for pediatric patients because it poses no risk of radiation, and pulmonary ultrasound has demonstrated clinical value in neonatal pulmonary disease [22-25]. Pulmonary ultrasound can be used for continuous real-time bedside monitoring of children with dyspnea, cyanosis, or other suspected lung diseases. It is especially suitable for neonatal ICU, since newborns must always remain in the incubator and their clinical conditions such as neonatal respiratory distress syndrome, meconium aspiration syndrome, and pulmonary hemorrhage change very quickly. It is hard to keep the children remain the same body position during the exam, therefore, as long as it is suitable for exposing the examination area, patients can be examined in any body position. For example, if the scan was started in a supine position but the child does not cooperate, scanning may be performed from the back.

\section{Requirements of Performing Fast and Accurate Ultrasound in ICU}

In a disease outbreak, more clinical staff may be needed to perform ultrasound examinations, especially in ICU. We think that a certified cardiologist or RPVI qualified vascular sonologist/technologist are wellpositioned to help train staff who are not familiar with ultrasound. With the basic knowledge and experience of ultrasound, the identification of lung diseases, pleural effusion, and other conditions will become more doable. In our team doctors with more than 3-year experience of basic cardiac ultrasound can quickly master the basis of lung ultrasound after 1 hour of training and can perform cross-sectional imaging after 1 day of training. Experts working in ICU have developed many diagnostic protocols and consensus to ensure the rapid and effective implementation of critical care ultrasound, such as the bedside pulmonary ultrasound (BLUE) process to identify different causes of dyspnea [26], the focused assessment with sonography for traumas (FAST) process [27] to detect hemoperitoneum, and the Rapid Ultrasound in Shock (RUSH) process $[28,29]$ to recognize distinctive shock etiologies in a short time. These protocols are used in emergent settings, and performing those types of ultrasound examination requires special training.

It is also recommended that people who have the experience of echocardiography should learn pulmonary ultrasound. Bataille et al. reported the diagnostic accuracy, sensitivity, and specificity of integrated cardiopulmonary ultrasound are higher than that of a single pulmonary ultrasound in the diagnosis of cardiogenic pulmonary edema and pneumonia, but not in the diagnosis of pulmonary embolism and pneumothorax [30]. Nazerain et al. reported that the sensitivity of diagnosis of an acute large pulmonary embolism (PE) can be significantly improved by simultaneously evaluating multiple organs (heart, lung, and lower extremity vein) compared to a single organ evaluation [31]. Here we presented our own case of diagnosing a large PE by multi-organ evaluation in Figure 6.
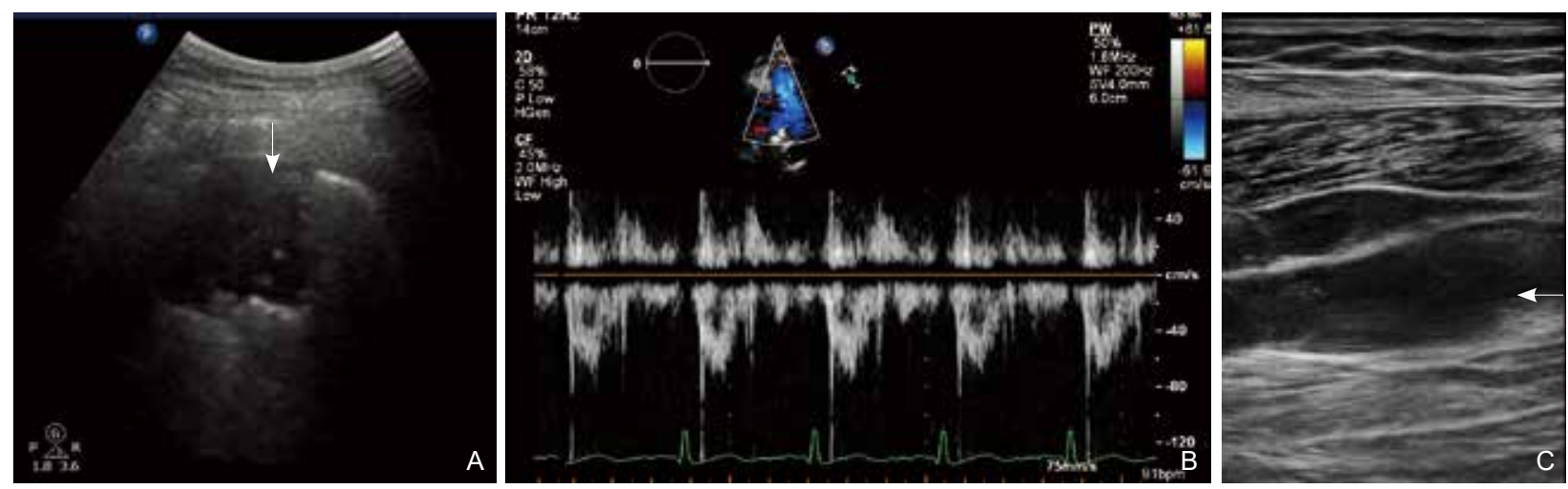

Figure 6 (A) A case of applying of multi-organ ultrasonography. A 72-year-old female suffered from cerebral infarction. She had dyspnea and decreased oxygen saturation on the second day after thrombolytic therapy. Ultrasonography found consolidation in two areas of lung field. (B) Echocardiography showed that there was notch on the blood flow spectrum of right ventricular outflow tract. (C) The right ventricular wall activity was abnormal, indicating pulmonary hypertension. Considering her medical history and suspicious cardio-pulmonary ultrasonography findings, the patient was most likely having pulmonary embolism. Then ultrasound of lower extremities verified the presence of thrombus in the femoral vein (arrow).

\section{Role of Critical Care Echocardiography}

One of the most common tasks of critical care ultrasound is to evaluate volume state and fluid responsiveness, since both insufficient and excessive fluid resuscitation lead to poor prognosis. Critical care echocardiography has the unique ability to screen for cardiac morphological and functional changes while providing a quantifiable probability of a patient's 
cardiovascular response to fluids. Common parameters used to describe cardiac function or fluid state include left ventricular end diastolic area (LVEDA), velocity of left ventricular outflow tract, the respiratory variation of the superior vena cava $(\triangle \mathrm{SVC})$, and the respiratory variation of the inferior vena cava $(\Delta \mathrm{IVC})[32-34]$. The images of our own experience of assessing volume state using $\triangle \mathrm{IVC}$ are shown in Figure 7.

Due to the "cross-talk" between the heart and other organs, acute kidney injury, respiratory failure, hypoxia, and systemic inflammation could all cause left or right heart dysfunction, or even whole heart failure [35]. Therefore, we recommend combining critical care ultrasound and echocardiography to monitor patients with cardiopulmonary dysfunction preceded by failure in other organs. Besides, focused critical care echocardiography is also useful in rapidly assessing more urgent cases, such as cardiac tamponade, acute left heart failure, acute right heart failure, and severe low blood volume state.
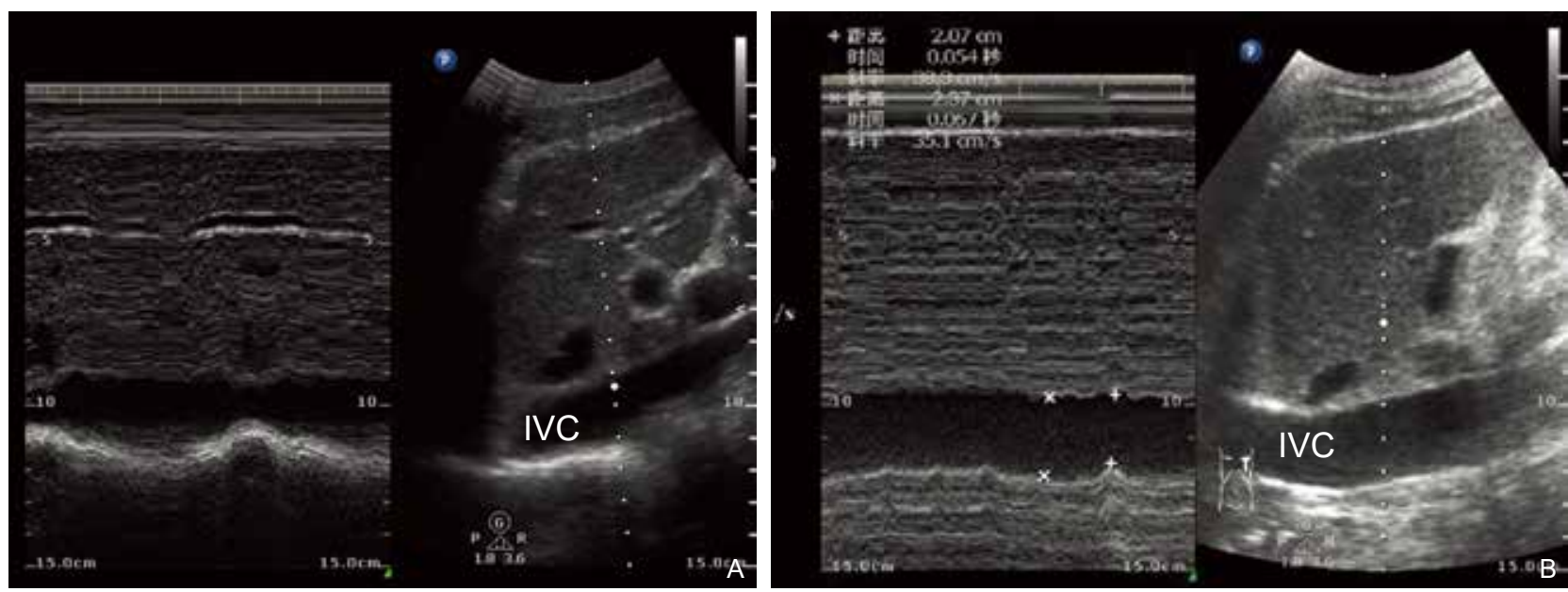

Figure 7 The image of inferior vena cava (IVC) is usually obtained by subxiphoid view or subcostal view in supine position. The diameter of IVC and its variability between inspiration and expiration can reflect the pressure of left atrium, which could further be used to evaluate the volume state and liquid responsiveness. Sample volume is usually taken at a distance of $2 \mathrm{~cm}$ from the right atrium entrance. The measurement is completed on M-mode sonography, to ensure the accuracy for identifying the breath phase. (A) Normal IVC with significant respiratory variability of inner diameter. (B) A patient on ventilator, and the IVC diameter is significantly widened with the respiratory variability reduced, indicating fluid overload.

\section{Role of Ultrasound in ECMO}

Patients with acute severe cardiac or pulmonary failure that is unresponsive to conventional management and potentially reversible should be initiated on extracorporeal membrane oxygenation (ECMO) [36]. Ultrasound plays a key role in the delivery and daily monitoring of ECMO. It not only guides the initiation of ECMO by identifying the intended vascular structure, but also help to prevent and detect some of the complications associated with ECMO [37].

\section{Role of Interventional Ultrasound in ICU}

Ultrasound has proven beneficial in guiding interventional procedures under a lot of circumstances. In the ICU, ultrasound-guided procedures can expedite waiting times and increase the safety, accuracy, and efficacy of many procedures. A number of ultrasoundguided procedures can be performed for critical care patients when necessary, including central venous catheter placement, catheterization and biopsy of inferior airway, paracentesis, thoracentesis, drainage of abscesses, and inferior vena cava filter placement, etc. [38]. Of note, when performing the interventional procedure, medical staffs always wear secondary or even tertiary personal protective equipment, which make the interventional operation even more complex. This is another great example of using ultrasound as a guide can significantly improve outcomes, shorten the operation time, and reduce occupational exposure.

\section{Ultrasound for Acute-On-Chronic Conditions}

Critical care ultrasound also plays a role of evaluating patients' basic diseases that could worsen their current condition, which is especially useful for elderly patients with chronic diseases, tumors, immunosuppression, and atypical inflammatory reaction. Critical care ultrasound also has flow-based recommendations for the screening for patients' basic diseases [16,39].

\section{Summary}

Ultrasound is an invaluable imaging modality in critical care to diagnose and guide the treatment of multiple life-threatening conditions including shock, respiratory failure, and multi-organ failure. The use of 
ultrasound in ICU has been shown to increase diagnostic accuracy, provide more efficient real-time monitor, and allow for more widespread, less expensive interventions for specific indications. In order to cope with the COVID-19 pandemic and provide better treatment for patients in need, clinicians should acknowledge and understand that a structured ultrasound training in critical care programs is of great importance.

\section{Conflict of Interest}

The authors have no conflict of interest to declare.

\section{References}

[1] Wu F, Zhao S, Yu B, Chen YM, Wang W, Song ZG, et al. A new coronavirus associated with human respiratory disease in China. Nature 2020; 579: 265-269.

[2] Zhou F, Yu T, Du R, Fan G, Liu Y, Liu Z, et al. Clinical course and risk factors for mortality of adult inpatients with COVID-19 in Wuhan, China: a retrospective cohort study. Lancet 2020; 395: 10541062.

[3] Guan WJ, Ni ZY, Hu Y, Liang WH, Ou CQ, He JX, et al; China Medical Treatment Expert Group for Covid-19. Clinical characteristics of coronavirus disease 2019 in China. $N$ Engl J Med 2020: NEJMoa2002032. DOI: 10.1056/NEJMoa2002032.

[4] Ksiazek TG, Erdman D, Goldsmith CS, Zaki SR, Peret T, Emery S, et al; SARS Working Group. A novel coronavirus associated with severe acute respiratory syndrome. N Engl J Med 2003; 348: 1953 66.

[5] Chacko J, Brar G. Bedside ultrasonography: Applications in critical care: Part I. Indian J Crit Care Med 2014; 18: 301-9.

[6] Vetrugno L, Bove T, Orso D, Barbariol F, Bassi F, Boero E, et al. Our Italian experience using lung ultrasound for identification, grading and serial follow-up of severity of lung involvement for management of patients with COVID-19. Echocardiography. 2020 Apr 1. DOI: 10.1111/echo.14664.

[7] Xu X, Yu C, Qu J, Zhang L, Jiang S, Huang D, et al. Imaging and clinical features of patients with 2019 novel coronavirus SARSCoV-2. Eur J Nucl Med Mol Imaging 2020; 47: 1275-1280.

[8] Tan W, Aboulhosn J. The cardiovascular burden of coronavirus disease 2019 (COVID-19) with a focus on congenital heart disease. Int J Cardiol. 2020 Mar 28. DOi: 10.1016/j.ijcard.2020.03.063.

[9] Shi H, Han X, Jiang N, Cao Y, Alwalid O, Gu J, et al. Radiological findings from 81 patients with COVID-19 pneumonia in Wuhan, China: a descriptive study. Lancet Infect Dis 2020; 20: 425-434.

[10] Lichtenstein D, Mézière G, Biderman P, Gepner A, Barré O. The comet-tail artifact. An ultrasound sign of alveolar-interstitial syndrome. Am J Respir Crit Care Med 1997; 156: 1640-6.

[11] Hasan AA, Makhlouf HA. B-lines: Transthoracic chest ultrasound signs useful in assessment of interstitial lung diseases. Ann Thorac Med 2014; 9: 99-103.

[12] Ding YQ, Bian XW. Analysis of coronavirus disease-19 (COVID-19) based on SARS autopsy. Zhonghua Bing Li Xue Za Zhi 2020; 49: 291-293. [In Chinese]. DOI: 10.3760/cma.j.cn112151-2020021100114.

[13] Buonsenso D, Piano A, Raffaelli F, Bonadia N, de Gaetano Donati K, Franceschi F. Point-of-care lung ultrasound findings in novel coronavirus disease-19 pnemoniae: a case report and potential applications during COVID-19 outbreak. Eur Rev Med Pharmacol Sci 2020; 24: 2776-2780.
[14] Thomas A, Haljan G, Mitra A. Lung ultrasound findings in a 64-yearold woman with COVID-19. CMAJ. 2020: cmaj. 200414. DOI: 10.1503/cmaj.200414. Epub ahead of print.

[15] Buonsenso D, Pata D, Chiaretti A. COVID-19 outbreak: less stethoscope, more ultrasound. Lancet Respir Med. 2020 Mar 20. DOI: 10.1016/S2213-2600(20)30120-X.

[16] Soldati G, Smargiassi A, Inchingolo R, Buonsenso D, Perrone T, Briganti DF, et al. Proposal for international standardization of the use of lung ultrasound for patients with COVID-19: a simple, quantitative, reproducible method. J Ultrasound Med. 2020 Mar 30. DOI: $10.1002 /$ jum.15285.

[17] Volpicelli G, Elbarbary M, Blaivas M, Lichtenstein DA, Mathis G, Kirkpatrick AW, et al; International Liaison Committee on Lung Ultrasound (ILC-LUS) for International Consensus Conference on Lung Ultrasound (ICC-LUS). International evidence-based recommendations for point-of-care lung ultrasound. Intensive Care Med 2012; 38: 577-91.

[18] Du J, Tan J, Yu K, Wang R. Lung recruitment maneuvers using direct ultrasound guidance: a case study. Respir Care 2015;60:e93-6.

[19] Bouhemad B, Brisson H, Le-Guen M, Arbelot C, Lu Q, Rouby JJ. Bedside ultrasound assessment of positive end-expiratory pressureinduced lung recruitment. Am J Respir Crit Care Med 2011; 183 341-7.

[20] Chiumello D, Mongodi S, Algieri I, Vergani GL, Orlando A, Via G, et al. Assessment of lung aeration and recruitment by ct scan and ultrasound in acute respiratory distress syndrome patients. Crit Care Med 2018; 46: 1761-1768.

[21] Caironi P, Carlesso E, Cressoni M, Chiumello D, Moerer O, Chiurazzi C, et al. Lung recruitability is better estimated according to the Berlin definition of acute respiratory distress syndrome at standard $5 \mathrm{~cm} \mathrm{H} 2 \mathrm{O}$ rather than higher positive end-expiratory pressure: a retrospective cohort study. Crit Care Med 2015; 43: 78190.

[22] Singh Y, Tissot C, Fraga MV, Yousef N, Cortes RG, Lopez J, et al International evidence-based guidelines on Point of Care Ultrasound (POCUS) for critically ill neonates and children issued by the POCUS Working Group of the European Society of Paediatric and Neonatal Intensive Care (ESPNIC). Crit Care 2020; 24: 65. DOI: 10.1186/s13054-020-2787-9.

[23] Kalafat E, Yaprak E, Cinar G, Varli B, Ozisik S, Uzun C, et al. Lung ultrasound and computed tomographic findings in pregnant woman with COVID-19. Ultrasound Obstet Gynecol. 2020 Apr 6. DOI: 10.1002/uog.22034.

[24] Moro F, Buonsenso D, Moruzzi MC, Inchingolo R, Smargiassi A, Demi L, et al. How to perform lung ultrasound in pregnant women with suspected COVID-19 infection. Ultrasound Obstet Gynecol. 2020 Mar 24. DOI: 10.1002/uog.22028.

[25] Abu-Rustum RS, Akolekar R, Sotiriadis A, Salomon LJ, Da Silva Costa F, et al. ISUOG consensus statement on organization of routine and specialist obstetric ultrasound services in the context of COVID-19. Ultrasound Obstet Gynecol. 2020 Mar 31. DOI: 10.1002/uog.22029.

[26] Lichtenstein DA. BLUE-protocol and FALLS-protocol: two applications of lung ultrasound in the critically ill. Chest 2015; 147: 1659-1670.

[27] Scalea TM, Rodriguez A, Chiu WC, Brenneman FD, Fallon WF Jr, Kato K, et al. Focused assessment with sonography for trauma (FAST): results from an international consensus conference. $J$ Trauma 1999; 46: 466-72.

[28] Perera P, Mailhot T, Riley D, Mandavia D. The RUSH exam: Rapid ultrasound in shock in the evaluation of the critically 11l. Emerg Med Clin North Am 2010; 28: 29-56, vii. 
[29] Keikha M, Salehi-Marzijarani M, Soldoozi Nejat R, Sheikh Motahar Vahedi H, Mirrezaie SM. Diagnostic Accuracy of rapid ultrasound in shock (RUSH) Exam; A systematic review and meta-analysis. Bull Emerg Trauma 2018; 6: 271-278.

[30] Bataille B, Riu B, Ferre F, Moussot PE, Mari A, Brunel E, et al. Integrated use of bedside lung ultrasound and echocardiography in acute respiratory failure: a prospective observational study in ICU. Chest 2014; 146: 1586-1593.

[31] Nazerian P, Vanni S, Volpicelli G, Gigli C, Zanobetti M, Bartolucci M, et al. Accuracy of point-of-care multiorgan ultrasonography for the diagnosis of pulmonary embolism. Chest 2014; 145: 950-957.

[32] Boyd JH, Sirounis D, Maizel J, Slama M. Echocardiography as a guide for fluid management. Crit Care 2016; 20: 274.

[33] Killu K, Coba V, Blyden D, Munie S, Dereczyk D, Kandagatla P, et al. Sonographic assessment of intravascular fluid estimate (SAFE) score by using bedside ultrasound in the intensive care unit. Crit Care Res Pract 2020; 2020: 9719751.

[34] Stawicki SP, Adkins EJ, Eiferman DS, Evans DC, Ali NA, Njoku $\mathrm{C}$, et al. Prospective evaluation of intravascular volume status in critically ill patients: does inferior vena cava collapsibility correlate with central venous pressure? J Trauma Acute Care Surg 2014; 76 : 956-63.

[35] Husain-Syed F, McCullough PA, Birk HW, Renker M, Brocca A, Seeger W, et al. Cardio-pulmonary-renal interactions: a multidisciplinary approach. J Am Coll Cardiol 2015; 65: 2433-48.

[36] Tsai HC, Chang CH, Tsai FC, Fan PC, Juan KC, Lin CY, et al. Acute respiratory distress syndrome with and without extracorporeal membrane oxygenation: a score matched study. Ann Thorac Surg 2015; 100: 458-64.

[37] Platts DG, Sedgwick JF, Burstow DJ, Mullany DV, Fraser JF. The role of echocardiography in the management of patients supported by extracorporeal membrane oxygenation. $J \mathrm{Am}$ Soc Echocardiogr 2012; $25: 131-41$.

[38] Nicolaou S, Talsky A, Khashoggi K, Venu V. Ultrasound-guided interventional radiology in critical care. Crit Care Med 2007; 35 S186-97.

[39] Peng QY, Wang XT, Zhang LN; Chinese Critical Care Ultrasound Study Group (CCUSG). Findings of lung ultrasonography of novel corona virus pneumonia during the 2019-2020 epidemic. Intensive Care Med. 2020 Mar 12: 1-2. DOI: 10.1007/s00134-020-05996-6. 